\title{
A general-purpose microcomputer configuration for controlling experiments
}

\author{
ELDON R. PARKS \\ Wabash College, Crawfordsville, Indiana 47933
}

\begin{abstract}
The circuitry required for connecting a KIM-1 microcomputer to devices in the experimental environment is shown together with an example of programming the microcomputer to control such devices. The experimental example involves sensory preconditioning of the nictitating membrane of the rabbit.
\end{abstract}

Microcomputers have several advantages over relay or solid-state modules for controlling experiments. They require no rewiring for the changes one may want to incorporate into an experiment. The "rewiring" is changed by changing the program. And, once the program is written, it can be kept stored on the shelf in an ordinary cassette, paper tape, or other storage device. Another advantage of microcomputers is they can read and store digital data directly, and, with circuits built from standard components outside the microcomputer, they can read and store analogue data. Finally, microcomputers can control more complex experiments than any reasonable number of relays or solid state programming modules, and do so in desk-top, sometimes briefcase-size, units.

\section{HARDWARE}

\section{The Microcomputer}

This paper is restricted to a discussion of the use of the microcomputer for controlling experiments and does not cover its use for data storage. The experimental example is one involving sensory preconditioning using the classically conditioned nictitating-membrane response of the rabbit. The microcomputer is the KIM-1, but most of what is said of the KIM-1 applies to other microcomputers as well.

The KIM-1 is inexpensive (\$245). It comes assembled on a single $8 \times 11$ in. board, which contains the microprocessing unit, two input-output (I/O) ports for communicating with the experimental environment, circuitry for sending information to and receiving information from both an ordinary cassette recorder and a Teletype, 1,110 bytes of programmable storage, a keyboard for entering programs into the microcomputer, and a seven-segment digital display for reading the contents of the microcomputer. In addition, the KIM-1 has a single-step function that is very useful for debugging programs. The unit has an interval timer and the $\mathrm{I} / \mathrm{O}$ ports are directly addressable.

The KIM-1 is an 8-bit machine; the words or data manipulated are 8 bits long. It uses the hexadecimal numbering system (i.e., numbers to the base 16, 0-9, and A.F). Thus the binary number 110 is equal to hexadecimal $C$ and the binary number 11111100 is equal to hexadecimal FC. Unless otherwise indicated by subscripting the base, the numbers used in this article are in hexadecimal.

For controlling experiments, switches must be turned on and off. Therefore, the job of the microcomputer is to send electrical signals to switches; the job of the person programming the microcomputer is to write programs that send these signals in the right order and at the right times.

Signals enter and leave the KIM-1 through two I/O ports: One is 8 bits, and the other is a $6-$ or 7 -bit port, depending upon the use made of the seventh. The 6-bit port is used to control the sensory-preconditioning experiment, and the 8-bit port is used to enter data. The discussion here will be restricted to the 6-bit port, which can be thought of as six wires or lines, each of which may be in either of two states: ready to receive or ready to send data. A voltmeter connected to any one of these six lines reads $5 \mathrm{~V}$ if the port is ready to receive data; it reads approximately 0 or $5 \mathrm{~V}$ (depending upon what the microcomputer has stored in the port) if it is ready to send data. In the KIM-1, any one of the lines is set to receive data by placing a zero in the corresponding position of memory Location 1703, and any line is set to send data by placing a one in the corresponding position of Location 1703. Thus, if Lines 1 and 5 receive data, and the remaining lines, $2,3,4$, and 6 , send data, the binary number 00101110 ( $2 \mathrm{E}$ in hexadecimal) is stored in 1703 . Since the present application calls for sending data from all six lines, the binary number 00111111 (3F) is placed into Location 1703, making them all output lines. Given the six are output lines, the voltage in each is determined by addressing memory Location 1702 . For example, if $0 \mathrm{~V}$ are desired on Lines 1 and 2, and $5 \mathrm{~V}$ on Lines 3.6, the binary number 00111100 is put into Location 1702 .

\section{External Hardware}

When the microcomputer is first turned on, it is designed to set the lines up for input in order to avoid turning on switches at random. Since all lines show 


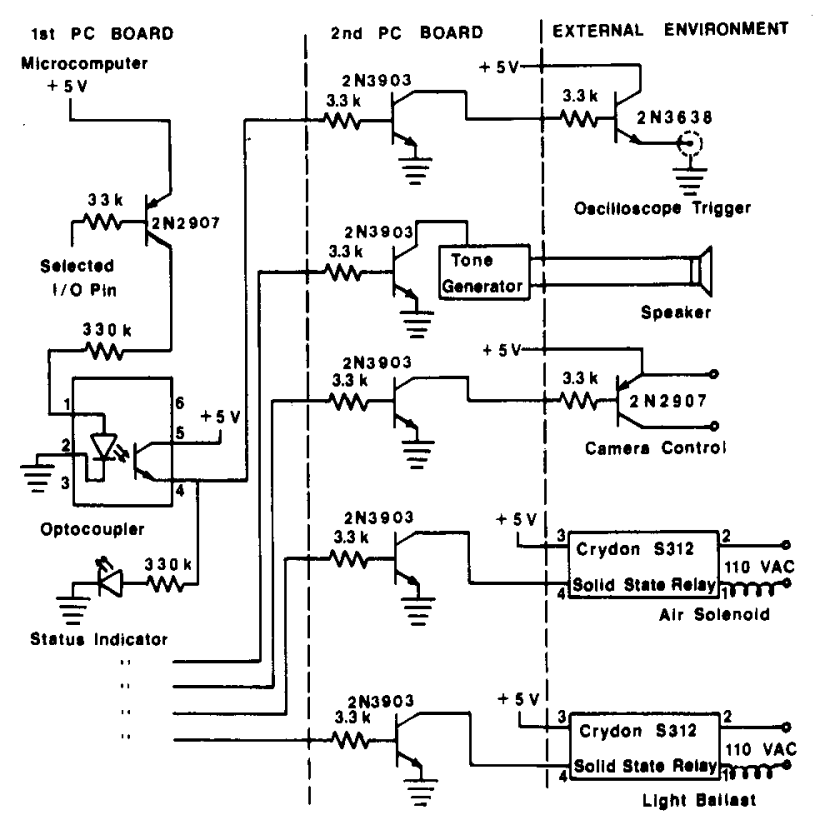

Figure 1. Circuitry external to the microcomputer. All $+5 \mathrm{~V}$, except the one labeled "microcomputer $+5 \mathrm{~V}$," come from the card-rack power supply. The circuits shown in the external environment are located near the units they control. All resistors are 1/4-W carbon; the optocoupler is a Fairchild FTK 0050. Only one of the six identical first-preconditioning board circuits is included in the figure, but five output pins from these circuits are shown.

$5 \mathrm{~V}$ when the $\mathrm{I} / \mathrm{O}$ ports are set for input, switching is done using zero voltages. Figure 1 shows the circuit for controlling switches with zero voltages, plus an optocoupler used to protect the microcomputer from the high voltages that might need to be switched in the experiment. When the particular $\mathrm{I} / \mathrm{O}$ line connected to the 2N2907 transistor is set to zero (GND voltage), current flows from the microcomputer's $+5 \mathrm{~V}$, through the emitter of the transistor, back through the $33 \mathrm{~K}$ resistor, to the $\mathrm{I} / \mathrm{O}$ line. This turns the transistor on, so that current flows from $+5 \mathrm{~V}$ through the $330-\mathrm{ohm}$ resistor, to the optocoupler. When the current is passed through the light-emitting diode (LED) in the optocoupler, it turns on the transistor connected between Pins 5 and 4 of the coupler. This transistor is then used to control a switch in the experiment. Eight of these circuits are placed on one $4 \times 4.5 \mathrm{in}$. Vector 4112.5 printed circuit board that is then plugged into a rack consisting of two aluminum strips onto which six R644 receptacles are attached. The receptacles are connected to the output lines of the microcomputer and are connected with each other in parallel. The status-indicator lights are mounted on the rack's front panel. These are unnecessary for control purposes, but are a great aid in programming and in determining which switches are on and which are off during an experiment. The card rack contains $+5-\mathrm{V}$ and $\pm 15-\mathrm{V}$ power supplies. Unless operational amplifiers are to be used, only $+5 \mathrm{~V}$ is needed.
A second $4 \times 4.5$ in. printed circuit board (Figure 1) contains six switching transistors, plus a 12-pin plug constructed of Emphenol microminiature strip connectors and wire-form contacts. Each pair of the 12 has one pin connected to the card rack $+5 \mathrm{~V}$ and one pin connected to the collector of the corresponding 2N3909 transistor. These six pairs of lines are fed out to: (1) an oscilloscope trigger, (2) a tone, (3) a light, (4) an air solenoid, and (5) a motorized Nikkon camera. The sixth is used to control a Hewlett-Packard frequency counter for data input.

Since ground voltages rather than $+5 \mathrm{~V}$ are being switched, and the oscilloscope trigger is already grounded, switching must be reversed (inverted) to trigger the oscilloscope. This is accomplished using the 2N3638 transistor. The camera is controlled using a 2N3909 transistor. The tone is produced by a 555 timer circuit also housed on the second printed circuit board (Since this is not a general-purpose tone generator, it will not be discussed.) Switching a tone generator using a fast relay (e.g., Grigsby-Barton 841 a) can be accomplished by going directly from one of the 2 N3909 transistors on the printed circuit board to the relay. The fluorescent light and air solenoid are controlled with solid state relays.

A list of the components and their costs for the KIM-1 system and card rack are given in Table 1 .

\section{SOFTWARE}

A Wernier-Orr diagram of the program controlling the sensory-preconditioning experiment is shown in Figure 2. Sensory preconditioning involves pairing two stimuli: in this case, tone-on, the first preconditioning stimulus $\left(\mathrm{PC}_{1}\right)$, and light-off $\left(\mathrm{PC}_{2}\right)$. The tone, $1,000 \mathrm{~Hz}$ at $78 \mathrm{~dB}$, is presented for $250 \mathrm{msec}$. It is followed by tone-on and light-off together for $100 \mathrm{msec}$, then light-off for $250 \mathrm{msec}$. These pairs are presented 15 times with a pseudorandom intertrial interval of from 50 to $70 \mathrm{sec}$ (mean $=60)$. Following the pairing of the two stimuli, an attempt is made to determine whether the animal has associated the two by conditioning a response to $\mathrm{PC}_{2}$ and testing to see whether the conditioning is transferred to $\mathrm{PC}_{1}$. In this example, the rabbits are given 60 conditioning trials on $\mathrm{PC}_{2}$ and 100 trials on $\mathrm{PC}_{1}$, with saving scores used as a measure of sensory preconditioning. Every 10th trial of conditioning is a test during which the unconditioned stimulus (3-psi air puff) is withheld and the camera photographing the nictitating-membrane response on the oscilloscope is activated.

Only the subroutine for straight, classical, nontest trial conditioning and the stimulus interval are shown in Figure 3, as an example of how to program an experiment. (A complete listing of the program is available from the author.)

This program uses only 7 of the available 56 instructions for the KIM-1 microcomputer: LDA, STA, 
Table 1

Parts and Price List for the KIM-1 and Card Rack

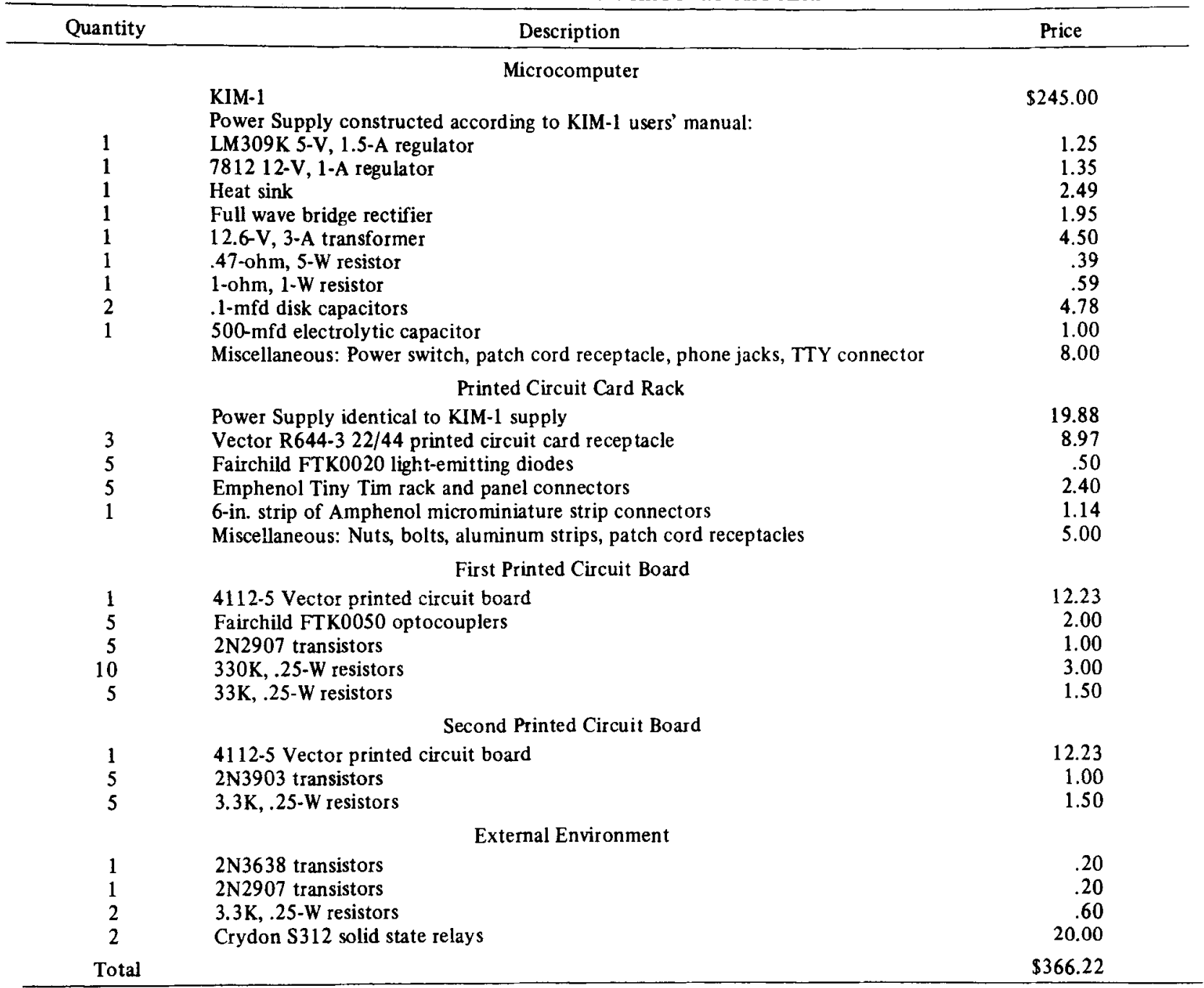

Note-Although not necessary for the sensory-preconditioning experiment, the KIM-1 has an add-on $4 K S$. D. Sales memory board (\$79.95) and $a \pm 15$-V power supply (\$14.15).

JSR, RTS, DEC, BNE, and BRK. The LDA (load accumulator) and STA (store accumulator) instructions are used to load data into memory. Steps 1 and 2 (LDA 07 and STA 1703) store 07 (binary 00000111) into Location 1703; I/O Lines 1,2 , and 3 are used as output lines. Steps 3-8 specify the number of trials to be given, and the intervals for which the conditioned stimulus (CS), unconditioned stimulus (UCS), and oscilloscope triggering will be activated. Steps 9.13 tum on the CS and oscilloscope by placing the binary number 11111100 (FC in hexadecimal) into the $\mathrm{I} / \mathrm{O}$ port at Location 1702 (the zeros in Positions 1 and 2 activate the corresponding switches) and keep those switches activated approximately $250_{10}$ msec by making $248_{10}$ jumps (JSR) to the millisecond timer at Step 25. Each jump to the timing subroutine takes 8 microsec; thus $8 \times 248=2 \mathrm{msec}$ is spent jumping. The number of jumps is controlled by the DEC (decrement the location specified by 1) instructions in Step 12 and BNE (branch if not zero) instruction in Step 13. If the value placed in Location 1796 is not yet zero, the microcomputer returns to Step 11 (labeled "JSR-1"). When the value in 1796 is finally decremented to zero (which it will after $248_{10}$ jumps), the microcomputer moves on to Step 14. Steps 14-18 turn on the CS and UCS for $100_{10} \mathrm{msec}$ and are the same type of loop as that between Steps 9 and 13. Steps 15-24 turn the switches off, cause a JSR to an ITI subroutine (which is a triplenested loop similar to the double loop in the millisecond interval timer), count the trials, branch back to Step 5 ("START") if the trial counter is not zero, and move on to BRK (break) to end the run if the trial counter does equal zero. The millisecond interval timer, Steps 25-33, is a double loop that counts down from 11 (Steps 29 and 30), then subtracts 1 from the 06 in Location 1798, reloads 27 into 1799, counts 11 down 


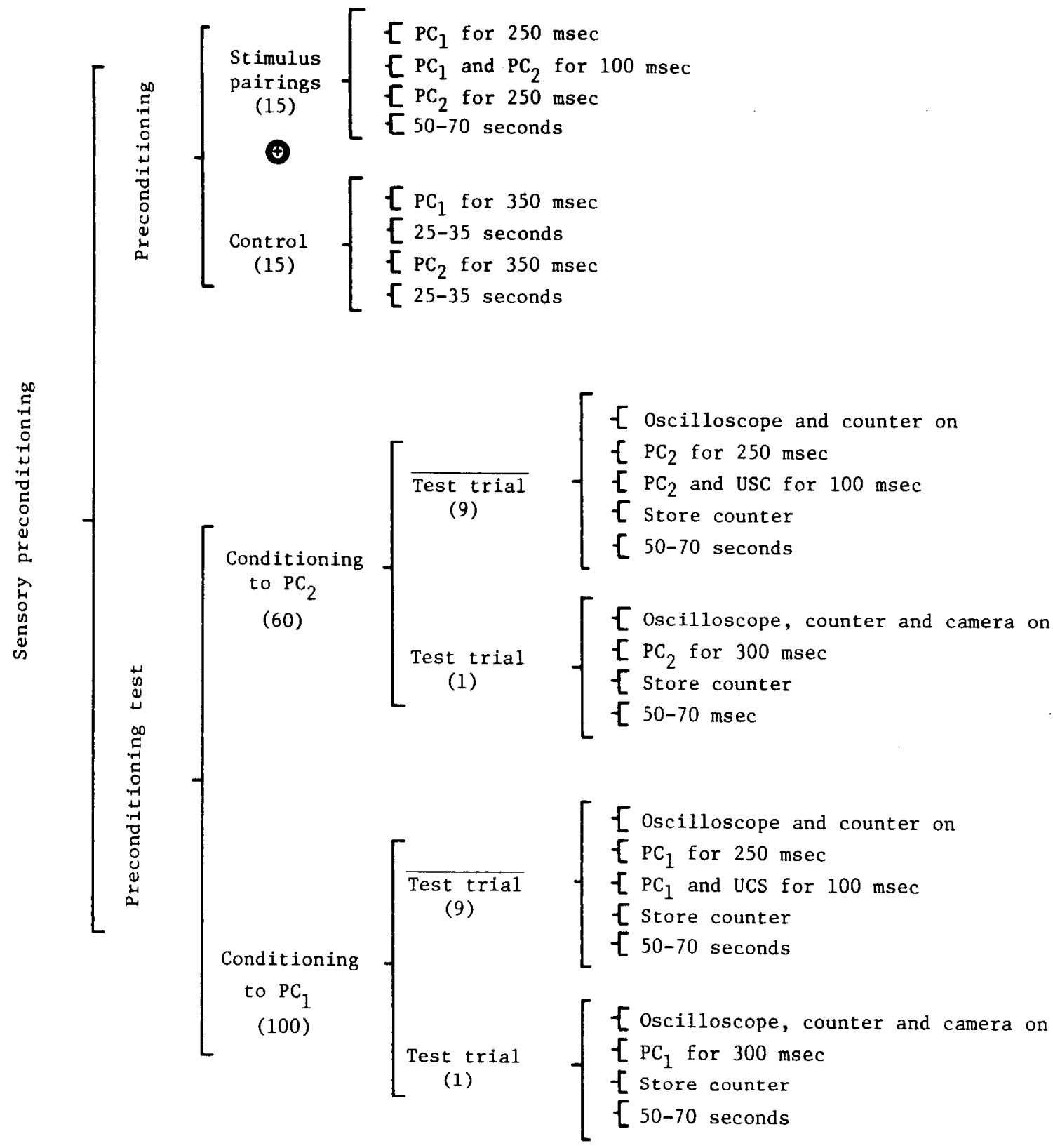

Figure 2. Wernier-Orr diagram of the program controlling the sensory preconditioning experiment. The $\oplus$ means "exclusive or." The line over "test trial" means "not." The numbers in parentheses indicate the number of times the operation is repeated, and the operations are listed in the sequence in which they occur. The advantage of the Wernier-Orr diagram is that the program is portrayed as nested subroutines, which is a logical way to program microcomputers. (For an introduction to Wernier-Orr diagramming, see Higgins, 1977.)

Figure 3

\begin{tabular}{|c|c|c|c|c|}
\hline Step & Label & $\begin{array}{l}\text { Mnemonic } \\
\text { OP Code }\end{array}$ & Operand & Comment \\
\hline 1 & & LDA & 07 & Sets Lines 1,2 , and 3 as output lines \\
\hline 2 & & STA & 1703 & \\
\hline 3 & & LDA & $3 \mathrm{C}$ & Number of trials, $60_{1}$ 。 \\
\hline 4 & & STA & 1795 & \\
\hline 5 & start & LDA & F8 & Number of milliseconds for CS and oscilloscope trigger, $248_{10}$ \\
\hline 6 & & STA & 1796 & \\
\hline 7 & & LDA & 63 & Number of milliseconds for CS and UCS, $99_{10}$ \\
\hline 8 & & STA & 1797 & \\
\hline 9 & & LDA & $\mathrm{FC}$ & \\
\hline 10 & & STA & 1702 & Puts GND voltage on Lines 1 and 2 \\
\hline
\end{tabular}




\begin{tabular}{llll}
11 & JSR-1 & JSR & Millisecond timer \\
17 & & DEC & 1797 \\
18 & & BNE & JSR-2 \\
19 & & LDA & 3F \\
20 & & STA & 1702 \\
21 & & JSR & ITI \\
22 & & DEC & 1795 \\
23 & & BNE & start \\
24 & & BRK & \\
25 & Millisecond timer & LDA & 06 \\
26 & & STA & 1798 \\
27 & begin & LDA & 11 \\
28 & & STA & 1799 \\
29 & DEC LC & DEC & 1799 \\
30 & & BNE & DEC LC \\
31 & & DEC & 1798 \\
32 & & BNE & begin \\
33 & & RTS & \\
\hline
\end{tabular}

Branches to "JSR-2" if value in 1797 is not 0

Turns off all switches

Jumps to random ITI subroutine (not shown)

Subtracts one from trial counter

Branches to "start" if trial counter is not 0

End

Loads high count (HC)

Loads low count (LC)

Subtracts one from low count

Branches to "DEC LC" if low count is not 0

Subtracts one from high count

Branches to "begin" if high count is not 0

Returns to program where it left off to enter timer

Figure 3. A program in mnemonic form for 60 nontest classical conditioning trials.

again, and so on, untill 11 has been counted down 06 times. This subroutine takes approximately $1 \mathrm{msec}$ to run, after which it returns (RTS) to the step following the one that directed the microcomputer to JSR to the subroutine. The time in milliseconds that the program remains in the millisecond interval timer subroutine is determined by the equation $\mathrm{HC}(9 \mathrm{LC}+14)+5$.

\section{SUMMARY}

The microcomputer described is used to run rabbits automatically in a sensory-preconditioning experiment. The only information the system requires before a run is whether the particular subject is in the stimuluspairing or in the control group. Once that information is loaded, all that is necessary is to push the "go" button and wait for the run to finish. The value of such a system is in the complexity of the experiment it can control, and that programs rather than wires are changed to alter an experiment.

A microcomputer, configured as described, is a general-purpose instrument in the sense that it can be used to control a wide variety of experiments. An addition of importance in many experimental applications is a connection to the microcomputer that causes it to respond to a switch (e.g., a Skinner box lever being closed in the experimental environment). This can be accomplished easily with a single 7400 NAND gate (for debouncing) connected to an interrupt pin of the microcomputer. Whenever the switch is closed, the microcomputer stops what it is doing and goes to the subroutines specified by the programmer. (See Gray, 1977 , for a discussion of debouncing switches.)

\section{REFERENCES}

Gray, E. W. How to "debounce" mechanical switches for for digital logic use. Popular Electronics, 1977, 8, 51-53.

Higgins. David A. Structured program design. Byte, 1977, 2. 146-151.

(Received for publication December 22, 1977; revision accepted January 9.1978. ) 